Claire MARTINOT

Modyco, CNRS, UMR 7114

Université Paris Descartes

45, rue des Saints-Pères- 75270 Paris cedex 06

cmartinot@free.fr

\title{
Reformulations paraphrastiques et stades d'acquisition en français langue maternelle
}

\section{Arrière-plan théorique : Paraphrase et reformulation}

Poser d'emblée que la maîtrise de l'activité paraphrastique est graduelle et qu'elle fournit un outil de repérage des stades d'acquisition en langue maternelle présuppose, d'une part, que cette activité participe du processus d'acquisition, d'autre part, que certaines paraphrases sont plus complexes, c'est-à-dire plus difficiles à produire que d'autres, soit parce que l'énoncé source (celui qui est paraphrasé) est « complexe », soit parce que la procédure paraphrastique qui s'applique à cet énoncé source met en œuvre plusieurs mécanismes simultanés.

$\mathrm{Si}$, comme le rappelle Fuchs (1982: 93), tout le monde s'accorde à reconnaître que la capacité au paraphrasage manifeste la maîtrise de la langue, il n'y a plus consensus quand il s'agit d'admettre que les relations de paraphrase sont inscrites dans la langue. Pour Fuchs (ibid.), les significations et les relations de paraphrase n'ont pas besoin d'être inscrites de façon stable en langue. Selon le même auteur, si elles l'étaient, cela aurait pour conséquence que le produit fini (c'est-à-dire la paraphrase d'un énoncé P) soit toujours le même.

Nous adoptons un autre point de vue. A la suite de Harris (1969, 1976, 1988, 1991), nous pensons que tout ensemble d'opérateurs a une transformée synonyme dans un ensemble moins contraint de ces opérateurs (Harris, 1976), autrement dit qu'il existe toujours, dans une langue naturelle, la possibilité de décrire un énoncé réduit par un énoncé analytique. Par ailleurs, l'ensemble des possibilités paraphrastiques d'une langue, qui correspondent aux quatre types d'équivalence suivants: équivalence sémantique, équivalence fonctionnelle, équivalence transformationnelle (avec ou sans restructuration ${ }^{1}$ ), équivalence descriptive (Ibrahim, 2007 : 1112) ne sont pas attestées, pour des ensembles équivalents de phrases, de la même façon, ni dans les mêmes proportions, d'une langue à l'autre, y compris dans des langues très proches comme l'italien et le français ainsi que la comparaison des reformulations définitoires (Gerolimich, Martinot, De Gioia, 2009) et la comparaison de la compétence paraphrastique (Martinot, Gerolimich, sous presse) chez des enfants francophones et italophones le montrent. Ce n'est donc pas l'activité paraphrastique des locuteurs qui «crée » les paraphrases. La possibilité plus ou moins étendue de paraphraser ou non une phrase donnée, que nous appellerons la plasticité paraphrastique, est inscrite dans chaque langue. Pensons par exemple à l'existence de plusieurs synonymes pour le verbe $\mathrm{X}$ en français et l'absence de synonyme pour son équivalent dans une autre langue. Ou encore, les possibilités de restructuration, qui dépendent du lexique et sont donc

- Nous remercions les relecteurs de cet article qui nous ont permis de préciser certains points.

${ }^{1}$ Mentionnées pour la première fois par Maurice Gross (1975 : 142-143) comme des transformations nouvelles, les transformations par restructuration permettent de relier des paires de phrases telles que : il semble que Marie travaille et Marie semble travailler, ou encore Paul admire les qualités de Marie et Paul admire Marie pour ses qualités. Elles ont ensuite trouvé un champ d'application important avec les travaux de Boons, Guillet, Leclère (1976), Guillet, Leclère (1992) où des phrases du type Max charge le camion de caisses sont reliées à des phrases de type : Max charge des caisses dans le camion. Les restructurations sont centrales dans l'explication des effacements (Boons (1971, Gross, 81), des grammaires locales (Ibrahim, 1979, 1984, 1997), de la grammaire des supports (Ibrahim, 2000a). Comme on le voit dans ces quelques exemples de restructuration, le lexique reste inchangé, et l'équivalence sémantique entre phrase de base et phrase restructurée est incontestable. 
spécifiques à chaque langue. L'énoncé source elle tenait par la main une petite fille est restructuré par les jeunes locuteurs francophones, dont nous parlerons plus bas, en :

a. elle tenait la main d'une petite fille

b. elle tenait la main à une petite fille

c. elle tenait une petite fille à la main

alors que, dans les mêmes conditions expérimentales, l'énoncé italien équivalent : teneva per mano una bambina ne se prête à aucune restructuration, tout simplement parce que le changement de préposition n'est pas possible en italien (Martinot, Gerolimich, sous presse).

La plasticité paraphrastique est déterminée par chaque langue. En revanche, la mise en œuvre du paraphrasage relève des locuteurs. L'activité paraphrastique s'inscrit selon nous dans une posture linguistique plus large que nous désignons par le terme de « reformulation » et qui n'implique pas nécessairement que l'énoncé reformulé soit équivalent sémantiquement à l'énoncé source, soit parce que le locuteur ne parvient pas à produire une équivalence soit parce qu'il veut dire autre chose. Nous avons défini la reformulation comme tout processus de reprise d'un énoncé antérieur qui maintient, dans l'énoncé reformulé, une partie invariante à laquelle s'articule le reste de l'énoncé, partie variante par rapport à l'énoncé source (Martinot, 1994). L'intérêt de cette nouvelle définition de la reformulation est de caractériser par un principe commun (l'articulation d'une partie invariante reprise à une partie variante inédite) des paires d'énoncés, énoncé source (ES) et énoncé reformulé (ER) qui vont de la répétition au changement de sens ${ }^{2}$ en passant par les différents types de paraphrases. Ainsi redéfinie, la reformulation permet de rendre compte d'une part d'un mode de production en langue, fréquent aussi bien chez les adultes que chez les enfants, non homogène, et donc difficile à décrire : rien n'oblige un locuteur à produire une reformulation de type : restructuration «pure ». La reformulation permet d'autre part de rendre compte d'une grande partie des procédures linguistiques d'acquisition et peut-être aussi de l'évolution des langues.

En conclusion, la production langagière se caractérise par une grande diversité dans la mise en œuvre de la plasticité paraphrastique, qui, elle, est inscrite dans la langue, mise en œuvre qui déborde largement la contrainte paraphrastique et que nous désignons par « reformulation ».

\section{Reformulations paraphrastiques et acquisition}

Notre propos se limitera toutefois aux reformulations paraphrastiques dans des productions enfantines recueillies lors d'un protocole expérimental que nous détaillerons plus loin.

On pourrait tout d'abord se demander - même si la réponse que nous apportons peut difficilement être démontrée - pourquoi les langues prévoient toujours plusieurs façons de dire la même chose et pourquoi les locuteurs semblent avoir besoin de formuler une même information de plusieurs façons que ce soit dans l'interlocution ou non.

Nous pensons que la production du sens linguistique se caractérise, entre autres choses qui ont été abondamment décrites, par le différentiel qui existe entre deux énoncés qui présentent un invariant sémantique. Comme si la reformulation paraphrastique, en apportant un éclairage différent sur une même information, complétait l'effet de sens, donnait plus de sens, sans pour autant saturer les potentialités signifiantes de cet énoncé. Du point de vue acquisitionnel, les reformulations paraphrastiques, contrairement aux reformulations qui modifient le sens ou à celles qui sont des répétitions, nous renseignent sur le savoir-faire linguistique que les enfants de

\footnotetext{
${ }^{2}$ Ce qui varie entre l'ES et l'ER répété est le statut différent de ER par rapport à ES, ER étant éventuellement prononcé par un autre locuteur; ce qui est invariant dans l'ER qui change le sens de l'ES est la structure identique entre l'ES et l'ER : ex. ES : Pierre regarde Marie > ER : Marie regarde Pierre.
} 
différents âges mettent en œuvre pour s'approprier le sens des énoncés sources qu'ils entendent et par conséquent peuvent être considérées comme un outil de repérage des stades d'acquisition. Ces procédures d'appropriation du sens sont nécessaires à l'acquisition de la langue.

\section{Protocole de recueil et mode d'analyse des données}

Les reformulations paraphrastiques que nous allons analyser proviennent d'un corpus recueilli auprès de 60 enfants francophones de 4, 6, 8 et 10 ans $^{3}$. Dans chaque tranche d'âge, 15 enfants tout venant sont enregistrés. Un adulte lit, une seule fois, l'histoire de Tom et Julie (cf. annexe) à chaque enfant puis l'enfant raconte à son tour la même histoire tout de suite après ${ }^{4}$.

L'histoire source est segmentée en 14 séquences narratives, ce qui permet d'apparier plus facilement les restitutions des enfants avec ces 14 séquences. La quasi-totalité des 14 séquences sont restituées par la quasi-totalité des enfants de 6 à 10 ans. A 4 ans, cinq séquences ne sont reformulées que par 4 à 9 enfants, les 9 autres séquences étant reformulées par la quasi-totalité des enfants de 4 ans.

Pour qu'une séquence source soit considérée comme reformulée, il faut qu'au moins une prédication complète de cette séquence soit présente, sous une forme ou une autre dans la production de l'enfant. Cette prédication est très souvent celle qui est la plus pertinente pour la suite de l'histoire :

\section{Ex. Séq.4.}

ES. Le lendemain matin, dans la cour de l'école, Tom guettait l'arrivée de sa nouvelle petite voisine. Dès qu'il l'a aperçue, il s'est dirigé vers la fillette et lui a tendu la boîte qu'il avait fabriquée pour elle, la veille.

ER. Marion (4 ans). et le lendemain matin la donna à Julie

Chaque production enfantine est donc segmentée en autant de séquences correspondant à celles $\mathrm{du}$ texte source. Il est alors possible de comparer une séquence source et une séquence reformulée. Cependant, ce niveau de segmentation, le plus souvent constitué de plusieurs prédications (cf. séq. 4 ci-dessus) ne permet pas de dire si l'enfant a répété, paraphrasé ou changé le sens de (toute) la séquence source. Pour répondre à cette question, qui fonde l'analyse des reformulations, telle que nous la faisons, le niveau de segmentation des paires d'énoncés analysés (ES-ER) doit être celui de la prédication simple (contenant un seul prédicat) comme dans les ES suivants :

Séq. 1. Elle tenait par la main une petite fille

Séq. 2. Julie sera ta voisine

ou de la prédication complexe (contenant deux prédicats) :

Séq. 2. Tom la place est libre à côté de toi (= la place qui est à côté de toi est libre)

Séq. 3. Tom était fou de joie à l'idée d'avoir peut être une nouvelle amie

Séq. 4. Tom guettait l'arrivée de sa nouvelle petite voisine

Nous avons donc extrait du texte source 13 prédications, simples ou complexes, ainsi que les reformulations paraphrastiques que les enfants en ont faites. Si les reformulations paraphrastiques

\footnotetext{
${ }^{3}$ Les enfants enregistrés ont 4;0 à 4;3 - 6;0 à 6;3 - 8;0 à 8;3 - 10;0 à 10;3.

4 Le même type de tâche a déjà été utilisé mais avec des objectifs très différents des nôtres qui testent par exemple les limites de la mémoire de travail à court terme (Ehrlich, 1994), les limites de la mémoire de travail à long terme (Ericsson, Kintsch, 1995), la mise en place de la cohérence locale ou globale à partir d'indices de la structure syntaxique de surface (Costermans, Fayol, 1997), ou la compréhension du texte (Denhière, 1984, Denhière, Rossi, 1991).
} 
augmentent en fonction de l'âge mais surtout si elles sont différentes d'un âge à l'autre, alors nous pourrons confirmer que la compétence paraphrastique est bien corrélée à la maîtrise de la langue 5 .

Précisons que la différenciation entre les paraphrases ne peut être un critère d'évaluation du degré d'acquisition de la langue qu'à condition d'admettre que certains énoncés sont plus complexes que d'autres, ce que permet le cadre théorique que nous avons évoqué en introduction, et qui prévoit qu'une langue soit constituée de phrases élémentaires et de phrases dérivées de ces phrases élémentaires.

\section{Analyse des reformulations paraphrastiques}

Les prédications que nous avons sélectionnées (cf. ci-dessous) dans l'histoire de Tom et Julie constituent un échantillon représentatif de divers types de complexité, auxquels nous avons adjoint quelques prédications simples. Si d'un point de vue syntaxique, les séquences ${ }^{6}: 1.2$ (relative explicative : prédication seconde) $; 2.1 ; 3 ; 4 ; 11$ (bien que la relative déterminative ne constitue pas une prédication seconde, la première proposition contient deux prédicats furent éblouis et la lumière, par ailleurs les relatives déterminatives sont encore plus difficiles à restituer que les relatives explicatives (Martinot et al, 2009), sans parler du passif et de la métaphore) ; 12 ; 13 (relative explicative : prédication seconde) sont des prédications complexes, du fait qu'elles renferment plus d'un prédicat, les autres séquences peuvent présenter d'aussi grandes difficultés lors de leur paraphrasage. Dans la séq. 1.1, les plus jeunes enfants omettent le complément prépositionnel par la main, ou modifient la préposition (par > dans), ce qui change le sens de l'énoncé reformulé par rapport à l'énoncé source. Dans la séq. 6.2, peu d'enfants ont réussi à rendre compte du même contenu sémantique concernant les informations temporelles et spatiales. Dans la séq. 10, de nombreux enfants ont modifié la construction de tourner sur luimême, ce qui entraîne un changement de sens. Dans la séq. 14, l'adjectif savant et encore plus l'adverbe extrêmement ont réservé des difficultés aux 4 ans qui ont produit peu de paraphrases.

Séq. 1.1. elle tenait par la main une petite fille

Séq.1.2. (elle tenait par la main une petite fille) que personne n'avait encore jamais vue

Séq. 2.1. Tom, la place est libre à côté de toi

Séq. 2.2. Julie sera ta voisine

Séq. 3. (3.1)Tom était fou de joie (3.2) à l'idée d'avoir peut-être une nouvelle amie

Séq. 4. Tom guettait l'arrivée de sa nouvelle petite voisine

Séq. 6.1. Julie chuchota à Tom

Séq. 6.2. je t'attends ce soir à $8 \mathrm{~h}$ sous le gros arbre à l'entrée de la forêt

Séq. 10. l'arbre était en train de tourner sur lui-même

Séq. 11. (11.1) Les enfants furent éblouis par la lumière (11.2) qui inondait l'intérieur de l'arbre

Séq. 12. Les fleurs semblaient se parler en chantant

Séq. 13. (Je veux apprendre à parler avec les oiseaux) qui savent tout ce qui se passe dans le ciel (avec les poissons ...dans l'eau, avec les fourmis ....sur la terre)

Séq. 14. Tom est devenu un enfant extrêmement savant

\subsection{Données quantitatives}

Les données chiffrées ne constituent jamais une explication en soi mais peuvent fournir une piste d'investigation. Ainsi si l'on regarde le total des séquences reformulées (ci-dessous), celles qui sont le plus fréquemment reformulées sous forme de paraphrases, tous âges confondus, sont les

\footnotetext{
${ }^{5}$ Nous avons de bonnes raisons de penser que l'activité paraphrastique détermine en grande partie l'acquisition parce que c'est à travers cette activité que le locuteur réussit plus ou moins à ajuster sa formulation à ce qu'il veut dire.

${ }^{6}$ Par commodité, nous appelons séquences les prédications qui sont extraites des séquences narratives du TS. Chaque «Séq » numérotée correspond en fait à une prédication.
} 
séquences 2.2 et 6.1. A l'inverse, les séquences les moins reformulées, sous forme paraphrastique, sont les séquences 10 et $11^{7}$.

Nombre de paraphrases pour chaque séquence
\begin{tabular}{|l|l|l|l|l|l|l|l|l|l|l|l|l|l|}
\hline & 1.1 & 1.2 & 2.1 & 2.2 & $\begin{array}{l}3.1 \\
\& 3.2\end{array}$ & 4 & 6.1 & 6.2 & 10 & $\begin{array}{l}11.1 \& \\
11.2\end{array}$ & 12 & 13 & 14 \\
\hline 4 ans & 4 & 1 & 2 & 4 & 0 & 1 & 4 & 0 & 0 & 0 & 1 & 0 & 2 \\
\hline 6 ans & 7 & 2 & 4 & 5 & 2 & 2 & 7 & 3 & 0 & 0 & 2 & 2 & 4 \\
\hline 8 ans & 9 & 6 & 7 & 12 & 7 & 3 & 12 & 2 & 0 & 0 & 3 & 3 & 9 \\
\hline 10 ans & 10 & 6 & 9 & 13 & 6 & 3 & 11 & 3 & 4 & 2 & 3 & 7 & 10 \\
\hline $\begin{array}{l}\text { Total des reformulations } \\
\text { paraphrastiques }\end{array}$ & 30 & 15 & 22 & 34 & 15 & 9 & 34 & 8 & 4 & 2 & 9 & 12 & 25 \\
\hline $\begin{array}{l}\text { Classement des énoncés en fonction du } \\
\text { plus grand nombre de ref.paraph. (1) } \\
\text { au plus petit nombre (10) }\end{array}$ & 2 & 5 & 4 & 1 & 5 & 7 & 1 & 8 & 9 & 10 & 7 & 6 & 3 \\
\hline
\end{tabular}

Le nombre de reformulations paraphrastiques augmente systématiquement et graduellement en fonction de l'âge avec un écart plus marqué (en moyenne) entre 6 et 8 ans $^{8}$.

\subsection{Outils d'analyse}

Nous présentons la synthèse des reformulations paraphrastiques attestées dans l'échantillon cidessus (voir 4) en suivant un ordre de "complexité » supposé croissant selon les résultats du tableau ci-dessus. Parallèlement aux résultats chiffrés, notre jugement de « complexité » résulte de l'analyse syntaxique des énoncés qui permet de distinguer les prédications simples des prédications complexes (ci-dessus. 3), mais aussi parmi les prédications simples, celles qui ont le statut de prédication seconde $(1.2 ; 13)$, celles qui ont plus d'un complément à droite du verbe $(1.1 ; 6.2)$, celles qui renferment un lexique complexe (chuchota est plus complexe que sera), celles qui contiennent un verbe opérateur ${ }^{9}$ (10). De la même façon, les prédications (syntaxiquement) complexes n'ont pas le même degré de complexité (= difficulté) : la séquence 1.2 devrait être moins difficile à paraphraser que les séquences 3,11 ou 12 , qui concentrent en même temps, des difficultés lexicales (métaphores), syntaxiques (opérateurs adjectival, nominal $^{10}$, verbal ; passif ; relative déterminative) et sémantiques (éventualité (3.2), unicité du procès alors qu'il y a deux verbes lexicaux (12). Tous ces critères concernent le plus ou moins grand degré de complexité supposé dans le TS. Mais les procédures de reformulation utilisées par les enfants (ou n'importe quel locuteur) sont aussi plus ou moins complexes, sachant que certains énoncés se prêtent - parce que la langue le permet - davantage à la paraphrase sémantique comme dans (a) qu'à des transformations par restructurations comme dans (b):

a.ES. Tom était fou de joie > ER. (6 ans) Tom était très heureux (séq. 3.1)

b.ES. Tom la place est libre à côté de toi > ER. (6 ans) Tom il y a une place à côté de toi (séq. 2.1)

\subsection{Reformulations paraphrastiques des prédications syntaxiquement simples}

${ }^{7}$ Rappelons que les données chiffrées ne concernent que les reformulations paraphrastiques et non le nombre total des reformulations qui, lui, nous renseignerait sur la perception qu'ont les enfants de la pertinence diégétique d'une information, éventuellement corrélée à la mémoire à court terme que gardent les enfants après l'audition de l'histoire.

${ }^{8}$ Les enfants qui n'ont pas produit de reformulations paraphrastiques ont répété la prédication source, en ont modifié le sens ou l'ont omise. Les répétitions et les omissions sont tendanciellement plus fréquentes à 4 ans que par la suite, les modifications du sens ont plutôt lieu à 6 et à 8 ans.

${ }^{9}$ Nous reprenons à Ibrahim (2000b) la définition de verbe opérateur : verbe à combinatoire presqu'entièrement libre entretenant une relation d'appropriation très faible ou nulle avec ce à quoi ils s'appliquent.

${ }^{10}$ Un opérateur adjectival ou nominal est un adjectif, un nom qui nécessite un argument : ex. content d'avoir une nouvelle amie - l'idée de partir à la campagne rendait Max euphorique. 


\subsection{1.}

Séq. 2.2. Julie sera ta voisine (34 reformulations paraphrastiques)

Séq. 6.1. Julie chuchota à Tom (34 ref. paraph.)

Ces deux prédications ont été le plus paraphrasées (parmi l'échantillon observé). Dans les deux cas, c'est entre les enfants de 6 et 8 ans que l'écart est le plus grand. La quasi-totalité des enfants a reformulé la séquence 2.2 par une paraphrase descriptive définitoire :

Adrien (4 ans) : la maîtresse lui dit : «mets-toi à côté de Tom !»

Antonin (8 ans) : «va t'asseoir à côté de Tom ! »

Les enfants décrivent le déplacement que doit faire Julie pour être la voisine de Tom. Or, cette description correspond à la définition analytique du mot voisine: Julie sera (l'enfant + l'élève) ${ }^{11}$ de la classe qui occupera pendant un certain temps la chaise (= s'assoira sur la chaise) qui se trouve à côté de celle de Tom (Ibrahim \& Martinot, 2004 ; Gerolimich, Martinot, De Gioia, 2009). Dès 6 ans, la définition analytique est attestée également sous une forme nominale :

Jean-Alexandre (6 ans) : tu as une nouvelle camarade à côté de toi

Marion (10 ans) : Julie sera ta camarade de classe

Dès 6 ans également, les enfants introduisent un nouveau point de vue (Jean-A. ci-dessus, Madeleine et Romain, ci-dessous) :

Madeleine (6 ans) : tiens il y a une place à côté de toi Tom je vais mettre Julie

Romain (10 ans) : la maîtresse mit Julie juste à côté de Tom

Dans la séquence 6.1, c'est également le mot prédicatif qui est l'objet d'une reformulation analytique définitoire (Ibrahim \& Martinot, 2004). Mais cette fois-ci, le verbe chuchota est défini dans l'immense majorité des cas, à 4 ans comme à 10 ans, par une définition analytique lacunaire $^{12}$. Les enfants reformulent chuchoter (= dire à voix chuchotée / basse) par dire :

Alexia (6 ans) : et un jour elle lui a dit d'ouvrir la boîte

A partir de 8 ans cependant, les reformulations paraphrastiques de cette prédication se diversifient par l'emploi d'un synonyme (dans le contexte de l'histoire) (a), par la restructuration du verbe chuchoter (b), par la description concrète du mouvement que fait l'enfant qui chuchote (c), par une forme de redondance qui manifeste l'analyse de chuchoter en dire, précédé de la façon de dire (d) et par la même analyse de chuchoter mais cette fois-ci, dire précède la façon de dire, supprimant ainsi la redondance (e) :

a. Maxima ( 8 ans) : un jour la petite fille souffla à son voisin : « ouvre la boîte »

b. Alexandre ( 8 ans) : et lui chuchota d'ouvrir la boîte

c. Camille (10 ans) : elle chuchote à l'oreille de Tom

d. Antonin ( 8 ans) : un jour chuchote et lui dit

e. Léo (10 ans) : Julie *disa à Tom en chuchotant

${ }^{11}$ Le signe + dans la parenthèse doit se lire « ou » : Julie sera l'enfant ou l'élève (...)

${ }^{12}$ Les reformulations analytiques définitoires lacunaires ou complètes sont étiquetées ainsi par référence aux matrices analytiques définitoires (MAD) (Ibrahim, 1997, 2010) qui sont des descriptions d'énoncés dans lesquels toutes les relations énonciatives et grammaticales sont explicitées, ce qui revient à déconstruire la grammaire. Les reformulations analytiques des enfants (ou même des adultes) ne correspondent jamais à ces MAD dans leur forme achevée mais à un état d'analyse partiel, par exemple ici, seul le verbe chuchoter sera décomposé et non pas l'ensemble de la prédication dans laquelle il est énoncé. 


\subsection{2.}

Séq. 1.1. elle tenait par la main une petite fille (30)

Séq. 14. Tom est devenu un enfant extrêmement savant (25)

L'écart le plus important dans les reformulations paraphrastiques de la séquence 1.1 se situe entre 4 et 6 ans et celles de la séquence 14, entre 6 et 8 ans.

La séquence 1.1 a été reformulée essentiellement de deux façons : soit par une réduction de la prédication à un complément prépositionnel en avec une petite fille :

Antoine (4 ans) : la maîtresse arriva en retard avec une petite fille

réduction qui disparaît quasiment à 10 ans, soit par une restructuration du verbe :

Camille (4 ans) : une maîtresse tenait la main d'une petite fille

Jean-Alexandre (6 ans) : la maîtresse tenait une petite fille à la main

Paul (10 ans) : elle tenait à la main une petite fille

Dès 6 ans, mais avec une progression quantitative entre 8 et 10 ans, sont attestés d'autres modes de reformulation: paraphrases sémantiques (a), équivalences transformationnelles (b), restructuration du verbe à la forme gérondive (c).

a.Rémy ( 6 ans) : elle ramenait une petite fille avec elle

Ninon (8 ans) : elle tenait par la main un enfant

Romain (10 ans) : elle tient par le bras une petite fille

b.Solène ( 8 ans $)$ : (...) qui tenait une petite fille par la main (permutation)

Léa (8 ans) : la maîtresse elle la tient par la main (pronominalisation)

c.Barbara (10 ans) : (...) en tenant une petite fille par la main

La séquence 14 a été paraphrasée par le même nombre d'enfants de 8 et 10 ans que la séquence précédente (1.1). En revanche, deux fois moins d'enfants de 4 et 6 ans ont paraphrasé la dernière prédication de l'histoire. Au niveau lexical, les difficultés qu'ont rencontrées les 4 et 6 ans concernent le verbe devenir (à 4 ans) et la restitution de l'adverbe extrêmement (4 et 6 ans) :

Adrien (4 ans) : Tom était savant

Alexia (6 ans) : Tom est devenu savant

A partir de 8 ans, la plupart des enfants reprennent le verbe devenir (pour lequel il n'y a pas de synonyme), expriment l'intensité contenue dans l'adverbe extrêmement par très ou la paraphrase suivante :

Antonin (8 ans) : il *connaissa tout il *devena un grand grand savant

Et à 10 ans, un enfant utilise l'adverbe source, et deux enfants expliquent ou définissent savant :

Camille (10 ans) : Tom *devena savant et intelligent

Théo (10 ans) : il devient très savant donc très intelligent

Presque tous les 10 ans suppriment un enfant qui correspond à une information redondante (50\% des 6 et 8 ans conservent ce nom).

\subsection{3}

Séq.6.2 je t'attends ce soir à 8 h sous le gros arbre à l'entrée de la forêt (8) 
Séq. 10. l'arbre était en train de tourner sur lui-même (4)

Les deux dernières prédications syntaxiquement simples présentent deux types de difficultés redoutables. La séquence 6.2 est construite à partir d'un verbe ne présentant aucune difficulté ni syntaxique ni sémantique. En revanche, la construction des compléments est fort complexe. Deux compléments réfèrent au temps ponctuel, les deux suivants à la localisation. Le second complément temporel précise le premier (ce soir), qui n'est pas nécessaire à l'information étant donné la situation d'énonciation. Les deux compléments locatifs sont en revanche nécessaires.

Tous les enfants ont exprimé au moins une information temporelle et une information locative mais comme le montre le tableau, très peu ont réussi à reformuler l'information complète équivalente à : $8 \mathrm{~h}$ (sous + auprès de + à) le (gros + grand) (arbre + chêne) (à l'entrée de + au début de + devant) la forêt.

Dès 4 ans, 3 enfants sur 5 ont restitué l'heure précise, tous les enfants plus âgés l'ont fait. La restitution de l'information locative complète a posé de nombreux problèmes du fait qu'il faut à la fois décrire l'arbre (2/5 l'ont fait à 4 ans, 7/12 à 6 ans, 6/12 à 8 ans, 10/13 à 10 ans) et le situer précisément par rapport à la forêt.

A l'entrée de la forêt a été reformulé par $3 / 5$ enfants de 4 ans par près du premier arbre $(E+$ de la forêt $)^{13}$, les autres ont simplement dit dans la forêt.

A 6, 8 et 10 ans, 3 enfants seulement de 6 et 8 ans, 4 de 10 ans ont répété à l'entrée de la forêt, ou ont paraphrasé par (au début + à la porte + devant) la forêt .

Pour résumer, à 4 ans, aucun enfant ne parvient à donner l'information complète, soit parce que l'heure précise manque, soit parce que la construction à l'entrée de la forêt ne fait pas sens (les énoncés les plus approchants étant : près du premier arbre de la forêt). De 6 à 10 ans, la mention temporelle indispensable est indiquée par tous les enfants mais très peu fournissent l'information relative et métaphorique que constitue l'entrée de la forêt :

Margot ( 6 ans) : Tom je t'attends à $8 \mathrm{~h}$ du soir près du grand arbre près de la forêt (changement de sens)

Ilan (8 ans) : rendez-vous ce soir à $8 \mathrm{~h}$ à l'arbre à la porte de la forêt (manque la description de l'arbre)

Léo $(10 \mathrm{ans})$ : ça disait à $8 \mathrm{~h}$ au grand chêne devant la forêt (OK : paraphrase fonctionnelle $\left.{ }^{14}\right)$

La séquence 10 a posé encore plus de problèmes de paraphrasage que la séquence précédente. Ce n'est qu'à 10 ans que 4/15 enfants parviennent à rendre compte de l'information exprimée par tourner sur lui-même autrement qu'en la répétant (cas relativement fréquent dès 4 ans) :

Théo (10 ans) : il pivote (paraphrase sémantique)

Marion (10 ans) : il pivote sur lui-même

Maxime (10 ans): et l'arbre fait des tours sur lui-même (reformulation définitoire analytique ou paraphrase descriptive)

Auparavant, les enfants (qui ne répètent pas) suppriment le complément prépositionnel (a) ou construisent un autre complément qui entraîne un changement de sens (b) :

a.Camille (4 ans) : l'arbre il tournait il tournait

b.Benjamin (6 ans) : l'arbre est en train de tourner autour d'eux

\subsection{Reformulations paraphrastiques des prédications syntaxiquement complexes \\ 4.4.1}

Séq. 2.1. Tom la place est libre à côté de toi (22 reformulations paraphrastiques)

${ }^{13}$ E note un élément effacé.

${ }^{14}$ Dans l'histoire, on admettra que arbre et chêne sont équivalents fonctionnellement (ou situationnellement). 
Nous avons considéré que cette prédication était complexe du fait qu'elle comporte une relative effacée disjointe de son antécédent: la place qui est à côté de toi est libre, que l'on peut réécrire en :

1.Il y a une place à côté de toi

2.Cette place est libre

La reformulation paraphrastique majoritaire et croissante de 6 à 10 ans est une restructuration de type :

Ninon (8 ans) : Tom il y a une place à côté de toi

Antonin ( 8 ans) : et il y avait une place libre à côté de Tom

qui n'est pas attestée à 4 ans et assez peu à 6 ans bien que cette construction soit très productive en français.

A 10 ans, un autre type de restructuration qui introduit un changement de point de vue est attesté chez deux enfants :

Barbara (10 ans) : vu que tu as une place à côté de toi (...)

\subsection{2}

Séq. 1.2. (...) que personne n'avait encore jamais vue (15)

Séq. 3. (3.1) Tom était fou de joie (3.2) à l'idée d'avoir peut-être une nouvelle amie (15)

Séq. 13. (je veux apprendre (...) oiseaux) qui savent tout ce qui se passe dans le ciel ${ }^{15}(12)$

Les prédications (1.2) et 13 sont des prédications secondes (relatives explicatives) respectivement situées en début et en fin d'histoire. Aucun enfant de 4 ans ne produit de paraphrase de la séquence 13 et un seul en produit pour la séquence (1.2). La prédication 13 est (probablement) plus complexe que la prédication 1.2 du fait que la relative contient une complétive : tout ce qui se passe dans le ciel. Mais ce n'est pas ce phénomène qui semble avoir réservé des difficultés aux enfants mais bien plutôt le fait que le sujet de savent n'est pas le même que le sujet du verbe précédent : je (Tom) veux apprendre à parler, puisque presque la moitié des enfants de 6 et de 8 ans ont modifié le sens de l'ES en disant :

Madeleine (6 ans) : je veux savoir parler avec les oiseaux pour savoir tout ce qui se passe dans le ciel

Les paraphrases de la prédication (1.2) se répartissent en trois types de paraphrases qui correspondent au groupe des 4-6 ans, au groupe des 8 ans et au groupe des 10 ans. Quantitativement, les 8 et les 10 ans fournissent trois fois plus de paraphrases que les plus jeunes. Les 4 et 6 ans produisent l'inverse d'une paraphrase descriptive en synthétisant la relative que personne n'avait encore jamais vue en un adjectif sémantiquement équivalent :

Sacha (6 ans) : ce matin la maîtresse tenait la main à une nouvelle élève

A 8 ans, la synthétisation de la relative se poursuit mais un nouvel adjectif apparait :

${ }^{15}$ La séquence source reprend trois fois de suite la même construction, d'abord avec les oiseaux qui savent tout ce qui se passe dans le ciel puis avec les poissons qui savent (...) dans l'eau et enfin avec les fourmis qui savent (...) sur la terre. Nous retenons la construction la mieux réussie chez chaque enfant, donc selon les cas, il s'agira des oiseaux, des poissons ou des fourmis. 
Océane (8 ans) : il y a une petite fille inconnue

D'autres enfants reformulent la relative sous la même forme mais avec un verbe ou un nom synonyme :

Rémy ( 8 ans) : (...) qu'on ne connaissait pas

Ninon ( 8 ans) (...) que les élèves n'avaient encore jamais vu

A 10 ans, plusieurs enfants modifient le point de vue tout en produisant une paraphrase fonctionnelle :

Maurine (10 ans) : c'est l'histoire d'une petite fille qui vient pour la première fois dans une école

Théo (10 ans) : c'est Julie qui entre dans une nouvelle école

Marie (10 ans) : un jour une nouvelle fille arrive dans une école

Maxime (10 ans) : il était une fois une petite fille qui faisait sa rentrée à l'école

Les paraphrases de la séquence 13 se répartissent en deux groupes. A 6 et 8 ans, les paraphrases sont pratiquement les mêmes: restructurations par effacement de la complétive ou par substitution de il y a à se passe, reprise du relatif dans quatre cas, substitution de parce que dans un cas :

Margot (6 ans) : avec les fourmis qui savent tout sur la terre

Antonin (8 ans) : aux oiseaux qui savent tout dans le ciel

Gaël (6 ans) \& Claire (8 ans) : aux oiseaux qui savent tout ce qu'il y a dans le ciel

Océane ( 8 ans) : aux oiseaux parce qu'ils savent tout dans l'air

A 10 ans, non seulement le nombre de reformulations paraphrastiques augmente nettement, mais en plus des restructurations par effacement de la complétive avec reprise du relatif (4 cas), il y a deux emplois de causatifs (parce que, car), l'un dans une paraphrase sémantique (savoir > connaître) avec effacement de la complétive, l'autre dans une restructuration avec il y a. Enfin, un enfant produit une restructuration complexe :

Maxime (10 ans) : je voudrais pouvoir parler aux poissons de tout ce qu'ils savent dans l'eau.

Le verbe parler est restructuré (parler avec les poissons > parler aux poissons de qch) puisqu'il acquiert un nouveau complément: le verbe savoir. Les oiseaux savent tout ce qui se passe devient parler de tout ce qu'ils savent. Dans l'ES, tout (ce qui se passe) est le complément de savoir, dans l'ER, le complément de savoir est aussi le complément de parler.

La prédication :

3.(3.1) Tom était fou de joie (3.2) à l'idée d'avoir peut-être une nouvelle amie (15 ref. paraph.)

a été aussi souvent reformulée et dans les mêmes proportions pour les quatre tranches d'âge que la prédication (1.2) (que personne n'avait encore jamais vue) que nous avons commentée au début de cette partie. La complexité syntaxique de ces deux prédications ne provient cependant pas des mêmes phénomènes. Si l'ensemble des enfants a reformulé le sentiment de très grande joie (3.1) à partir de 6 ans (un enfant de 4 ans a fait de même) :

Camille (4 ans) : il est très content

M (6 ans) : Tom était très heureux

Léa (8 ans) : Tom était ravi 
assez peu d'enfants ont paraphrasé l'ensemble de la séquence 3 ( 2 enfants de 6 ans, 7 de 8 ans et 6 de 10 ans). Parmi ces enfants, la construction de l'adjectif opérateur (fou de joie à...) paraphrasée le plus souvent par très/tout content de, augmente nettement entre 6 et 8 ans. La construction du nom opérateur à l'idée de est reformulée en revanche par un seul enfant de 8 ans :

Marc (8 ans) : Tom était ravi parce qu'il croyait qu'il allait se faire ami

La construction complexe (à l'idée d'avoir...) est reformulée plus souvent à 10 ans (3 enfants) :

Marion (10 ans) : Tom il est tout content à l'idée qu'il pourra se faire peut-être une nouvelle camarade de classe

Léo (10 ans) : Tom était très content il pensait avoir peut être une nouvelle amie

A partir de 8 ans, quelques enfants expriment l'éventualité de la nouvelle relation amicale entre les enfants (Marc, ci-dessus) :

Anthony ( 8 ans) : content de pouvoir avoir une nouvelle amie

Maurine (10 ans) : Tom est très content d'avoir une nouvelle voisine et peut-être sa nouvelle copine

Les difficultés de paraphrasage de la séquence 3 tiennent davantage à la succession de 2 constructions complexes (adjectif opérateur s'appliquant à un nom opérateur) qu'à l'interprétation de cette séquence. On peut classer ces difficultés dans l'ordre suivant (du plus accessible au moins accessible) :

Reformulation de l'ensemble, à partir de 6 ans

Reformulation paraphrastique avec adjectif opérateur, à partir de 6 ans

Reformulation paraphrastique de l'éventualité, avec verbe opérateur, à partir de 8 ans

Reformulation paraphrastique de l'éventualité avec le même adverbe, à partir de 10 ans

Reformulation paraphrastique avec nom opérateur, à partir de 10 ans

\subsection{3}

Les deux prédications suivantes ont été paraphrasées exactement dans les mêmes proportions pour chaque tranche d'âge (et au total par 9 enfants) :

Séq. 4. Tom guettait l'arrivée de sa nouvelle petite voisine (9 reformulations paraphrastiques)

Séq. 12. Les fleurs semblaient se parler en chantant (9)

Il faut préciser que le petit nombre de reformulations paraphrastiques, mais aussi de reformulations en général, de ces deux prédications est dû à la faible valeur diégétique de chacune de ces prédications (voir le TS en annexe).

La double prédication de la séquence 4 (guettait \& l'arrivée) a été paraphrasée de trois façons différentes : à 4 ans, l'enfant reprend le verbe et donne un synonyme de l'arrivée (paraphrase sémantique) :

Laurana (4 ans) : le lendemain matin Tom guettait l'entrée de sa voisine 
A 6 et 8 ans, les enfants analysent le verbe guetter par une reformulation définitoire lacunaire dans laquelle ils ne reprennent que l'un des deux verbes descriptifs de la matrice analytique définitoire, attendre ${ }^{16}$ :

Madeleine (6 ans) : le lendemain il attendait Julie

Un autre enfant de 6 ans tente une analyse plus complète en transformant guettait en adverbe :

Daphné (6 ans) : une fois dans la cour il attendait *guettement sa petite voisine

Dans les deux cas, les enfants ont effacé le nom prédicatif.

A 8 ans, les trois enfants qui paraphrasent cette prédication définissent toujours de façon lacunaire guetter par attendre, mais deux d'entre eux rétablissent le nom prédicatif et pour l'un sous la forme d'une restructuration :

Maxima (8 ans) : un petit garçon qui attendait une nouvelle voisine à l'entrée

A 10 ans, deux enfants analysent guetter toujours de façon lacunaire mais en le reformulant par le verbe voir modalisé :

Alexandrine (10 ans) : et il essaye de la voir

Océanie (10 ans) : il voit fixement la petite fille (paraphrase très approximative, le verbe regarder est évidemment plus approprié)

La séquence 12 :

Séq. 12. Les fleurs semblaient se parler en chantant

présente deux difficultés d'ordre différent, la première se trouve dans la compréhension ou dans la reformulation paraphrastique de l'opérateur semblaient (aucun enfant de 4 ans n'a repris sous une forme ou une autre ce verbe), et la seconde dans la compréhension de la relation sémantique qui unit parler et chanter. Les enfants doivent comprendre qu'il n'y a pas deux procès mais un seul : les fleurs se parlent, procès que les fleurs réalisent en chantant. L'opérateur s'applique d'ailleurs à la modalité (en chantant). Pour décider si les enfants avaient paraphrasé ou non cette prédication, nous n'avons tenu compte que de la relation entre parler et chanter qui constituent la partie prédicative de la séquence 12 (se parler: prédication principale, en chantant: prédication seconde).

Peu de variations paraphrastiques sont disponibles : l'enfant de 4 ans a inversé les deux verbes (ce qui ne change pas le sens étant donné la signification de chacun des verbes, à comparer avec marcher en boitant, nager en crawlant, skier en godillant ...), un enfant de 8 ans a remplacé parler par discuter :

Alexandre (8 ans) : (...) où on sentait qu'il y avait des fleurs qui discutaient en chantant

Les autres enfants ont repris les verbes (se) parler et chanter. La reformulation du verbe opérateur sembler présente davantage de variations et permet aussi de distinguer des étapes acquisitionnelles pour l'emploi de ce verbe. Aucune tentative de reformulation à 4 ans. A 6 ans, deux tentatives dont une seule produira une paraphrase avec parler et chanter (cf. Chloé) :

${ }^{16}$ On admettra que guetter quelqu'un se définit de façon analytique par : regarder fixement dans une direction précise en attendant quelqu'un, ou encore attendre quelqu'un en regardant fixement dans une direction précise. 
Alexia(6 ans) : on dirait que les fleurs elles chantaient

Chloé (6 ans) : ils ont l'impression que les fleurs elles se parlent en chantant

A 8 ans, les tentatives de restituer semblaient par un synonyme sont nombreuses (cf. aussi Alexandre, plus haut), mais seules Charlotte et Claire (ci-dessous) ont fourni une équivalence sémantique de semblaient sans pour autant paraphraser l'ensemble de la prédication :

Charlotte (8 ans) : c'est comme si les fleurs ils parlaient ensemble (non paraph.)

Claire ( 8 ans) : des fleurs qui paraissaient chanter (non paraph.)

Solène ( 8 ans) : on disait même qu'elles parlaient en chantant (paraphrase)

Antonin ( 8 ans) : on croyait que les fleurs se parlaient en chantant (paraphrase)

A 10 ans, sur trois enfants qui paraphrasent la séquence 12 (autant qu' à 8 ans), deux parviennent aussi à fournir un synonyme de sembler :

Barbara et Léo (10 ans) : on/il avait l'impression que les fleurs se parlaient en chantant

\subsection{4}

La dernière prédication de notre analyse :

Séq. 11. Les enfants furent éblouis par la lumière qui inondait l'intérieur de l'arbre (2 reform. paraph.)

est la moins paraphrasée de tout l'échantillon. Dans son ensemble, elle n'a été paraphrasée que par deux enfants de 10 ans, et encore Léo n'a pas reformulé les enfants furent éblouis :

Paul (10 ans) (...) les enfants éblouis par la lumière qui sortait du tronc

Léo (10 ans) (...) à l'intérieur c'est inondé de lumière

Cette séquence concentre plusieurs phénomènes généralement considérés comme complexes : le passif, la double prédication de la première partie de la phrase : les enfants furent éblouis du fait qu'il y avait une lumière qui (...), la relative déterminative, la métaphore de la lumière qui inonde un endroit et renvoie à une très grande quantité de lumière (l'intensité de cette lumière est évoquée par être éblouis).

A 4 et 6 ans, aucune reformulation n'évoque le contenu sémantique de la relative. Les deux enfants de 4 ans qui reformulent une partie de la séquence 11 n'évoquent que l'existence de la lumière, sans son intensité :

Pauline (4 ans) : il y avait de la lumière

Camille (4 ans) : ils ont vu de la lumière

A 6 ans, les deux enfants reprennent le verbe au passif mais sans complément d'agent, ce qui fait que le participe passé éblouis fonctionne davantage comme un adjectif que comme un verbe (d'autant que l'auxiliaire est à l'imparfait dans un cas, au présent dans l'autre) :

E (6 ans) : (...) les enfants étaient éblouis

Chloé (6 ans) : (...) ils sont éblouis parce qu'il y a la lumière

La relation causative entre l'existence de la lumière et l'éblouissement des enfants, qui en est la conséquence, apparaît cependant chez Chloé. On peut penser que cette relation causative doit être comprise sinon produite avant que les enfants ne produisent un passif complet (avec complément d'agent). 
A 8 ans, les enfants ne paraphrasent toujours pas l'ensemble de la prédication mais les trois enfants qui reformulent la première partie de cette prédication introduisent tous les trois soit un indice de grande quantité de lumière, indice qui provient du verbe métaphorique inonder de la relative, soit un indice de grande intensité (Charlotte), qui provient du verbe être éblouis, et un enfant produit un passif complet (Antonin) :

Léa ( 8 ans) : et ça s'est ouvert avec des lumières

Antonin ( 8 ans) : ils étaient éblouis par tant de lumière

Charlotte (8 ans) : il s'est ouvert il y a eu un espère de + un éclairement

A 10 ans, deux enfants sont parvenus à paraphraser la séquence 11, l'un a cependant supprimé la métaphore (Paul), l'autre n'a pas évoqué la conséquence (ils furent éblouis) de la grande quantité de lumière (Léo) :

Paul (10 ans) : les enfants éblouis par la lumière qui sortait du tronc

Léo (10 ans) : à l'intérieur c'est inondé de lumière (...)

La forme passive est complète chez Paul. Léo a restructuré la relative déterminative. Nous considérons que ce type de restructuration est particulièrement complexe d'autant que Léo conserve la métaphore.

Les autres enfants de 10 ans n'ont pas paraphrasé l'ensemble de la séquence mais deux d'entre eux ont produit une paraphrase transformationnelle de la première partie de la séquence par une translation (Marc) et par une transformation à la forme active (Alexandrine) :

Marc (10 ans) : (...) il y a eu une lumière éblouissante

Alexandrine (10 ans) : (...) une grande lumière l'éblouit

Un troisième enfant a tenté de reformuler la métaphore mais le résultat aboutit à un changement de sens. Dans l'ES, la lumière a les propriétés d'un liquide (évoquées par inonder), dans l'ER, c'est l'élément liquide qui a les propriétés de la lumière :

Océanie (10 ans) : l'arbre s'ouvre et ils voient une mer éblouissante (non paraph.)

Malgré le très faible nombre de paraphrases à 10 ans, la différence entre les enfants de 8 et de 10 ans se manifeste ici par la production de métaphores à 10 ans, par une restructuration complexe du verbe métaphorique, par deux paraphrases transformationnelles, par un nombre sensiblement plus grand d'enfants qui établissent une relation de causalité entre la lumière et l'éblouissement.

\section{Conclusion}

Notre premier objectif était de montrer que la capacité à paraphraser s'accroît en fonction de l'âge, au cours de la période des acquisitions tardives (à partir de 4 ans).

$\mathrm{Au}$ vu des résultats quantitatifs portant sur le nombre de reformulations paraphrastiques d'un échantillon représentatif de prédications extraites d'une histoire à restituer, une réponse positive ne fait aucun doute. On peut ajouter que cette capacité se diversifie également en fonction de l'âge.

Notre deuxième objectif était de montrer que la capacité paraphrastique des enfants ne s'applique pas de la même façon sur des prédications décrites comme simples ou comme complexes par la littérature. Là encore, les résultats quantitatifs sont nets. Quatre des six prédications syntaxiquement simples (séq. 2.2, 6.1, 1.1, 14) sont celles qui ont été le plus souvent paraphrasées, y compris par les 4 ans. A l'inverse, parmi les six prédications le moins souvent 
reformulées se trouvent quatre prédications syntaxiquement complexes (séq. 11, 4, 12, 13), que les 4 ans n'ont pratiquement pas paraphrasées.

Ce résultat confirme (Martinot, et al, 2009) qu'au cours du processus d'acquisition, les enfants reformulent d'abord les prédications syntaxiquement simples et ensuite seulement les prédications syntaxiquement complexes. Cela signifie que, d'une certaine manière, les enfants les distinguent mais aussi que les deux types de prédication sont inscrits dans la langue.

Le nombre total de reformulations par séquence (34 à 2) suit globalement un ordre de complexité croissante (1 à 10), évalué à partir de différents phénomènes: nombre de prédicats par prédication, nombre de constituants par prédication simple, statut des prédications (principale ou seconde), nombre d'opérateurs, degré de complexité sémantique : verbes complexes (chuchoter), certains adverbes, certaines notions comme l'éventualité (peut-être), la description d'une perception (semblaient), l'expression de la modalité lexicale (parler en chantant), certaines métaphores (la lumière inondait l'intérieur de l'arbre ou l'entrée de la forêt sont complexes, d'autres comme fou de joie le sont beaucoup moins). La combinaison de ces phénomènes dans la même prédication accroît évidemment la complexité de cette prédication. Parmi les prédications syntaxiquement simples (parce qu'elles ne contiennent qu'un seul prédicat et qu'elles sont ellesmêmes prédications principales) : (6.2) je t'attends ce soir (...), (10) l'arbre (...) tourner sur luimême, la grande difficulté qu'ont rencontrée les enfants lors de la restitution et a fortiori quand ils ont tenté de fournir un équivalent sémantique tient dans le premier cas à l'accumulation d'informations dont la pertinence doit être repérée (6.2) et dans le second cas à la construction réflexive du verbe tourner sur lui-même (10).

Notre troisième objectif était de rechercher si les procédures de reformulation paraphrastiques sont différentes d'un âge à l'autre, ce qui pourrait laisser supposer que certaines procédures sont plus complexes que d'autres.

Les reformulations paraphrastiques descriptives, qui consistent à analyser un mot prédicatif de la séquence source, sont un bon indicateur de la simplicité vs complexité telles que l'enfant les perçoit. Dès 4 ans, les enfants sont capables de décrire le sens d'un mot quand il n'est pas complexe (voisine). S'il est complexe, alors la reformulation définitoire est lacunaire (chuchoter > dire) d'abord puis devient de plus en plus complète entre 8 et 10 ans (cas attestés avec: chuchoter, guetter, tourner sur lui-même, savant, extrêmement).

Les paraphrases sémantiques qui consistent à reformuler le nom ou le verbe source par un synonyme sont attestées à partir de 6 ans mais le lexique ne se diversifie (à travers les différentes reformulations) qu'à partir de 8 ans. De même, l'emploi de noms opérateurs et prédicatifs est attesté à partir de 8 ans.

Les paraphrases transformationnelles, procédures les moins courantes relativement aux paraphrases sémantiques, descriptives et fonctionnelles, sont attestées à 8 ans (permutation) et 10 ans (translation, passif > actif).

Les restructurations qui sont une sous-classe des paraphrases transformationnelles en ce qu'elles sont contraintes par le lexique sont attestées dès 4 ans pour certaines (restructuration du verbe tenir par la main une petite fille, restructuration de la place est libre). Les réductions ou effacements sont également précoces (relative : que personne n'avait encore jamais vue > nouvelle; complétive : savent tout ce qui se passe dans le ciel > savent tout du ciel). En revanche, la restructuration d'un verbe conjugué en un gérondif (qui transforme donc ce dernier en prédicat second: en tenant par la main), la restructuration qui reformate une prédication complexe précédée d'une prédication simple (parler avec les poissons qui savent tout ce qui se passe dans l'eau > parler aux poissons de tout ce qu'ils savent dans l'eau) en une seule prédication tout aussi complexe, et la restructuration d'une relative déterminative contenant une 
métaphore (la lumière qui inondait l'intérieur de l'arbre > à l'intérieur c'est inondé de lumière) ne sont attestées que chez quelques enfants de 10 ans.

Enfin les paraphrases fonctionnelles qui modifient le point de vue (Julie sera ta voisine > je vais mettre Julie à côté de toi) ne sont pratiquement pas présentes avant 10 ans.

Pour conclure, les reformulations paraphrastiques définitoires qui sont des paraphrases descriptives sont présentes à tous les âges. Il est probable qu'elles ont un rôle particulièrement important à jouer dans la construction du sens mais peut-être aussi dans l'élaboration des paraphrases sémantiques et des restructurations, ces deux procédures devenant plus nombreuses en fonction de l'âge. Les paraphrases fonctionnelles sont en revanche les plus tardives. Elles correspondent à une plus grande maîtrise du système énonciatif de la langue mais aussi à une aptitude à introduire une équivalence entre l'énoncé source et l'énoncé reformulé, non prévue par la langue.

\section{Références}

Boons J-P 1971, « Métaphore et baisse de la redondance », Langue française 11, 15-17.

Boons J-P., Guillet A., Leclère C.

Costermans, J. \& Fayol, M.

1976, La structure des phrases simples en français, Genève : Droz.

1997, Processing interclausal relationship. Mahwah, Lawrence Erlbaum Associates.

Denhière, G. 1984. Il était une fois ...Compréhension et souvenir de récits. Lille : Presses Universitaires de Lille.

Denhière, G. \& Rossi, J-P. (eds.).

1991. Text and text processing. Amsterdam : Elsevier Science Publishers, North-Holland.

Ehrlich, M-F. 1994. Mémoire et compréhension du langage. Lille : Presses Universitaires de Lille.

Ericsson, K.A \& Kintsch, W.

1995. «Long-term working memory”. Psychological Review 87, 215-251.

Fuchs C. $\quad$ 1982, La Paraphrase, Paris : PUF.

Gerolimich, S., Martinot, C., De Gioia, M.

2009, «Reformulations définitoires spontanées chez des francophones et des italophones de 6, 8 et 10 ans », Autour de la définition 6, http://publifarum.farum.it/

Gross M. 1975, Méthodes en syntaxe, Paris : Hermann.

Gross, M. $\quad$ 1981, «Les bases empiriques de la notion de prédicat sémantique », Langage 63, 7-52.

Guillet A., Leclère C.

1992, « Restructuration du groupe nominal », Langages 63, 99-128.

Harris Z.S. 1969, «The two systems of Grammar: Report an Paraphrase », Transformation and Discourse Analysis Papers 79 [612-92 in Papers]

Harris Z.S. $\quad$ 1976, Notes du cours de syntaxe, Paris : Seuil.

Harris Z.S. $\quad$ 1988, Language and Information, New York : Columbia University Press.

Harris Z.S. $\quad$ 1991, A Theory of Language and Information : A Mathematical Approach, Oxford/New York : Clarendon Press.

Ibrahim A.H. 1979, Etude comparée des systèmes verbaux de l'arabe égyptien, de l'arabe moderne et du français, Doctorat d'Etat, université Paris 7.

Ibrahim A.H. 1984, «Sur le statut de quelques accidents syntactico-sémantiques », in Pierre Attal et Claude Muller (éds.), De la syntaxe à la pragmatique: Linguisticae Investigationes Supplementa, vol.8, Amsterdam/Philadelphia : John Benjamins Publishing Company, 241259.

Ibrahim A.H. 1997, «Pour une définition matricielle du lexique », Cahiers de lexicologie 71.

Ibrahim A.H. 2000a, «Constantes et variables de la grammaire des supports dans quelques langues Romanes », De la grammaire des formes à la grammaire du sens, Tübingen : Max Niemeyer Verlag, 241-251. 
Ibrahim A.H. 2000b, «Une classification des verbes en six classes asymétriques hiérarchisées », in Françoise Cordier, Jacques François, Bernard Victorri (éds.), Sémantique du lexique verbal : Syntaxe et sémantique 2, Presses universitaires de Caen, 81-98.

Ibrahim A.H. 2007, «Introduction », La langue et l'information, (Trad. française de Language and Information, Harris Z.S, 1988), Paris : Cellule de Recherche en Linguistique, 3-26.

Ibrahim A.H. 2009, «Les conditions de la prédication dans les langues » in Ibrahim A.H, Prédicats, prédication et structures prédicatives, Paris : Cellule de Recherche en Linguistique, 1249.

Ibrahim A.H. 2010, "Supports d'actualisation et dualité constitutive de la prédication », in Ibrahim A.H, Supports et prédicats non verbaux dans les langues du monde, Paris : Cellule de Recherche en Linguistique, 36-73.

Ibrahim A. H. \& Martinot, C.

2004, «Les reformulations matricielles lacunaires des enfants », Travaux de Linguistique $48,21-40$.

Martinot C. 1994, La reformulation dans des productions orales de définitions et explications. (Enfants de maternelle), Thèse de Doctorat, Dir. Blanche-Noëlle Grunig, Université Paris VIII.

Martinot C., Gerolimich S., Paprocka-Piotrowska U., Sowa M.

2008, «Reformuler pour acquérir sa langue maternelle ? Investigation auprès d'enfants français, italiens et polonais de 6, 8 et 10 ans », in Schuwer M, Le Bot M.-C., Richard E. (éds.) : Pragmatique de la reformulation, Presses Universitaires de Rennes, 221-240.

Martinot, C., Kuvac-Kraljevic, J., Bosnjak-Botica, T., Chur L.

2009, «Prédication principale vs seconde à l'épreuve des faits d'acquisition», in Ibrahim A.H, Prédicats, prédication et structures prédicatives, Paris: Cellule de Recherche en Linguistique, 50-81.

Martinot C., Gerolimich S.

(Sous presse), «Acquisition de la compétence paraphrastique par des enfants francophones et italophones de 4 à 10 ans », in la Synonymie au-delà du lexique, éd. Forum, Université de Udine.

\section{Annexe}

\section{Tom et Julie}

1) Ce matin-là, la maitresse est arrivée dans la cour de l'école plus tard que d'habitude. Elle tenait par la main une petite fille que personne n'avait encore jamais vue.

2) Arrivée en classe, la maîtresse a dit : « Les enfants, je vous présente votre nouvelle camarade, elle s'appelle Julie. Tom, la place est libre à côté de toi, Julie sera ta voisine, sois bien gentil avec elle !»

3) Tom était fou de joie à l'idée d'avoir peut être une nouvelle amie. Le soir, chez lui, il a fabriqué une petite boîte ronde, rouge et dorée, pour Julie.

4) Le lendemain matin, dans la cour de l'école, Tom guettait l'arrivée de sa nouvelle petite voisine. Dès qu'il l'a aperçue, il s'est dirigé vers la fillette et lui a tendu la boîte qu'il avait fabriquée pour elle, la veille.

5) Julie aimait tellement cette boîte qu'elle la prenait toujours avec elle. Quand la maîtresse disait: «Sortez vos affaires !», Julie posait délicatement la boîte entre Tom et elle, sur leur table de travail.

6) Un jour, Julie chuchota à Tom : « Ouvre la boîte ! » Tom souleva le couvercle et découvrit un morceau de papier sur lequel Julie avait écrit : " Je t'attends ce soir à 8h, sous le gros arbre, à l'entrée de la forêt ».

7) Tom avait un peu peur parce qu'il lui était interdit d'aller dans la forêt, surtout la nuit.

8) Mais à $8 \mathrm{~h}$ du soir, il était tout de même au rendez-vous, Julie l'attendait déjà.

9) Sans dire un mot, la petite fille prit la main de Tom et frappa 3 fois sur le tronc du gros arbre.

10) Au bout de quelques minutes, les enfants entendirent un grincement. L'arbre était en train de tourner sur luimême.

11) Tout à coup, le tronc s'ouvrit et les enfants furent éblouis par la lumière qui inondait l'intérieur de l'arbre. Ils firent quelques pas et l'arbre se referma derrière eux 
12) Tom et Julie se trouvaient dans un jardin merveilleux où les fleurs semblaient se parler en chantant. Alors Julie dit à Tom : «Viens, traversons le jardin, il y a une grande fête pour toi, ce soir. Jusqu'à minuit, tu as le droit de demander à notre Roi tout ce que tu veux ».

13) Tom a répondu : «Je veux apprendre à parler avec les oiseaux qui savent tout ce qui se passe dans le ciel, avec les poissons qui savent tout ce qui se passe dans l'eau et avec les fourmis qui savent tout ce qui se passe sur la terre ».

14) Et depuis ce jour, Tom est devenu un enfant extrêmement savant.

\title{
Résumé
}

\begin{abstract}
Mots-Clés : Paraphrases, reformulations, prédications simples et complexes, complexité syntaxique, acquisitions tardives, français langue maternelle.
\end{abstract}

L'acquisition de la maîtrise paraphrastique est aussi inexplorée, dans le champ de la recherche sur l'acquisition de la langue maternelle, qu'incontournable pour l'enfant de 4 ans environ, qui est encore très loin d'être parvenu à la maîtrise achevée de sa langue maternelle. Cet article a pour objectif de montrer que les paraphrases produites par des enfants d'âges différents nous renseignent sur les stades successifs d'acquisition, évalués à partir du différentiel qualitatif que l'on peut décrire entre un énoncé source et un énoncé reformulé sous forme paraphrastique.

Le corpus est recueilli auprès de 60 enfants (15 enfants de 4, 6, 8 et 10 ans) qui ont restitué, immédiatement après l'avoir écoutée une fois, une histoire d'environ 500 mots.

Un échantillon représentatif de 13 prédications simples (contenant un seul prédicat) et complexes (contenant deux prédicats) a été sélectionné dans le texte source et comparé systématiquement à toutes les reformulations paraphrastiques produites par tous les enfants.

Les résultats de l'analyse linguistique montrent que la capacité paraphrastique s'accroît et se diversifie en fonction de l'âge. Selon le type de complexité, syntaxique et/ou sémantique, de l'énoncé source, mais aussi selon sa potentialité à être paraphrasé, la difficulté n'est pas la même. Enfin, le résultat le plus important montre que les procédures de reformulations paraphrastiques sont attestées tendanciellement selon l'ordre suivant: paraphrases descriptives, puis sémantiques, puis transformationnelles et enfin fonctionnelles.

\section{Paraphrastic Reformulations and Stages in the Acquisition of French as a mother Tongue.}

\section{Key-words :Paraphrases, reformulation, simple and complex predications, syntactic complexity, late} Language acquisition, French as a mother Tongue.

The acquisition of paraphrastic mastery is conspicuous by its absence in surveys in the field of research on the acquisition of the mother tongue, a skill that is indispensable for the child around the age of 4 years who is still a long way from having achieved a mastery of her mother tongue. This article aims to show that these paraphrases, produced by children of a range of ages, tell us about the different stages of acquisition, assessed by the qualitative differences that can be described between a source statement and one that has been reformulated in its paraphrastic form.

The study group consisted of 60 children (in groups of 15 aged 4, 6,8 and 10 years) who asked to recount a story of 500 words immediately after having heard it told to them once.

A representative sample of 13 simple (containing a single predicate) and complex phrases (containing two predicates) was selected from the source text and systematically compared to the full set of paraphrastic reformulations employed by all the children.

The results of the linguistic analysis show that the paraphrastic capacity increases and becomes more diverse with age. Depending upon the type of complexity, either syntactic and / or semantic, the difficulty varies according to the source statement, but also on the basis of its potential for being paraphrased. Finally, the most important result demonstrates that paraphrastic reformulation procedures are encountered in the following order: descriptive paraphrases, then semantic, followed by transformational and, finally functional ones. 